\title{
Making the brain young to treat depression
}

Antidepressant treatment combined with psychotherapy is known to be more effective for the treatment of depression than either treatment approach alone. The neurobiological basis of this effect, however, is unknown. While antidepressants affect neurotransmitters, research has not supported the idea that they treat depression simply by correcting chemical imbalances in the brain.

The most common treatment approach for depression is a combination of antidepressants, such as fluoxetine (commonly known as Prozac), and exposure therapy. This type of therapy can be modeled in rodents through extinction training. Although this training initially makes adult rodents less fearful of a conditioned stimulus, after some time the fear responses will return upon re-exposure to the fearinducing stimulus. In contrast, juvenile mice experience permanent erasure of fear memories after extinction training.

Fluoxetine has been shown to enhance neuronal plasticity in the hippocampus and the cortex. Researchers in Eero Castrén's lab at the University of Helsinki (Finland) asked whether the drug similarly affects fear networks in the brain and whether this effect underlies its antidepressant action. The fear-conditioning and extinction paradigm was used in adult mice to investigate whether fluoxetine might reactivate juvenile-like plasticity in the fear-conditioning network. Adult mice that were given fluoxetine while they went through extinction training behaved much like young mice: their fear responses were extinguished faster, and recovery and renewal of their fear responses were attenuated (Science 334, 1731-1734; 2011).

Next, the scientists investigated the underlying causes of the enhanced extinction and found that fluoxetine shifted neurons in the amygdala, a brain area critical for fear learning and extinction, toward an immature state, thereby reactivating juvenile-like plasticity in the fear-conditioning network. As a result, communication between neurons in the amygdala was also enhanced. The researchers also found that the drug increases levels of BDNF, a chemical implicated in fear extinction, in the amygdala. They confirmed that this chemical was essential to the effect by showing that fluoxetine failed to prevent

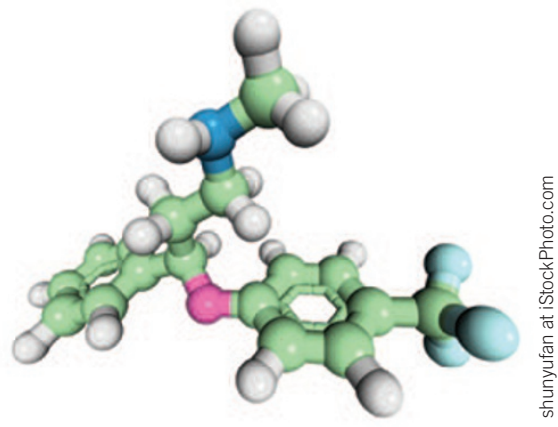

renewed freezing a week after extinction in mice lacking a copy of the gene encoding BDNF.

These results suggest that the drug gives mice the renewed plasticity needed to make or break new connections. This may prime the adult brain to reshape neural networks in response to new behavioral experiences, such as extinction training, allowing the animal to learn that a oncefrightening stimulus is now harmless. These findings may explain why drug treatments need to be combined with therapy for the optimal treatment of depression, anxiety and post-traumatic stress disorder.

Kara Rosania

\section{SILENCED GENES TURNED ON TO TREAT ANGELMAN'S SYNDROME}

No effective therapies exist for Angelman's syndrome, a neurodevelopmental disorder that can cause seizures, sleep disorders, speech problems and motor control issues. Patients do not express a functional copy of the enzyme ubiquitin-ligase E3A (UBE3A), which marks old proteins in the brain for breakdown. As a result, proteins accumulate in the brain, interfering with neuronal function.

In rodents and humans, neurons express UBE3A only from the maternal copy because translation of the father's copy of the gene is repressed. The disorder is therefore the result of mutations or deletions in the maternal copy of the gene. People with Angelman's syndrome have a normal copy of the gene from their fathers, suggesting that 'un-silencing' the dormant paternal copy in neurons might prevent the disorder.

A group of researchers led by Benjamin D. Philpot at the University of North Carolina (Chapel Hill, NC) tested this idea. Using cortical neurons from mutant mice that were genetically engineered to fluoresce when UBE3A was turned on, they screened for molecules that could turn on the functional paternal copy of the gene. The screen identified several molecules that were able to 'un-silence' the father's copy of the gene in these cells (Nature doi:10.1038/nature10726; published online 21 December 2011).

The most promising of these drugs was topotecan, which is approved for the treatment of cancer in humans. In living transgenic mice that modeled the disease, the drug caused the father's copy of the gene to be expressed in neurons in several areas of the brain as well as in the spinal cord. The degree to which the gene was 'un-silenced' was correlated with the concentration of the drug in the brain. They also confirmed that the protein encoded by the 'un-silenced' gene functioned normally in the neurons. Further, a single course of treatment with topotecan could permanently modify expression of UBE3A. The scientists suggest that other diseases in which a functional copy of a gene is silenced could be targeted using a similar approach.

Topotecan is a promising treatment for Angelman's syndrome in humans because it is currently approved for use in adult and pediatric cancer patients, and it has a demonstrated ability to penetrate into the central nervous system. The researchers next need to test for off-target effects and to determine an optimum dosage of the drug.

Kara Rosania 